\title{
Simulation of an Electromagnetic Acoustic Transducer Array by Using Analytical Method and FDTD
}

\author{
Yuedong Xie, ${ }^{1}$ Sergio Rodriguez, ${ }^{1}$ Wenbo Zhang, ${ }^{1}$ Zenghua Liu, ${ }^{2}$ and Wuliang Yin' \\ ${ }^{1}$ School of Electrical and Electronic Engineering, University of Manchester, Manchester M60 1QD, UK \\ ${ }^{2}$ College of Mechanical Engineering and Applied Electronics Technology, Beijing University of Technology, Beijing 100124, China \\ Correspondence should be addressed to Wuliang Yin; wuliang.yin@manchester.ac.uk
}

Received 4 June 2015; Accepted 9 November 2015

Academic Editor: Gyuhae Park

Copyright (c) 2016 Yuedong Xie et al. This is an open access article distributed under the Creative Commons Attribution License, which permits unrestricted use, distribution, and reproduction in any medium, provided the original work is properly cited.

\begin{abstract}
Previously, we developed a method based on FEM and FDTD for the study of an Electromagnetic Acoustic Transducer Array (EMAT). This paper presents a new analytical solution to the eddy current problem for the meander coil used in an EMAT, which is adapted from the classic Deeds and Dodd solution originally intended for circular coils. The analytical solution resulting from this novel adaptation exploits the large radius extrapolation and shows several advantages over the finite element method (FEM), especially in the higher frequency regime. The calculated Lorentz force density from the analytical EM solver is then coupled to the ultrasonic simulations, which exploit the finite-difference time-domain (FDTD) method to describe the propagation of ultrasound waves, in particular for Rayleigh waves. Radiation pattern obtained with Hilbert transform on time-domain waveforms is proposed to characterise the sensor in terms of its beam directivity and field distribution along the steering angle, which can produce performance parameters for an EMAT array, facilitating the optimum design of such sensors.
\end{abstract}

\section{Introduction}

There are a variety of nondestructive testing (NDT) techniques employed in industries, such as magnetic particle inspection (MPI), electromagnetic methods (EM), eddy current methods, and ultrasonic methods [1-7]. Due to its advantages of having good penetration depth and mechanical flexibility, the piezoelectric ultrasonic method is widely used for thickness measurement, flaw evaluation, and material characterization [8-13]. The transducer frequently used is made of piezoelectric ceramics or crystals [12-15]. However, one primary disadvantage of the piezoelectric ultrasonic testing is the need to have good sonic contact with the test piece, typically by means of a couplant for acoustic impedance matching [16].

Electromagnetic acoustic transducers (EMATs) are becoming increasingly popular due to their noncontact nature $[17,18]$. An EMAT sensor typically consists of a permanent magnet providing a large static magnetic field and a coil carrying an alternating current which is placed next to the test piece [19-21]. There are two EMAT interactions which can produce ultrasound: magnetostriction for magnetic materials and the Lorentz force mechanism for conducting metallic materials $[18,20,22]$. Because an EMAT generates ultrasonic waves directly into the testing piece instead of coupling through the transducer, an EMAT has advantages in applications where surface contact is not possible or desirable [23, 24]. Another attractive feature of EMAT which is a variety of waves modes can be produced based on different combinations of coils and magnets $[18,25]$. In this paper, only EMAT based on Lorentz force mechanism to generate surface waves is discussed.

Considerable works have been reported on EMAT modelling [26-30]. All of these papers divide EMAT modelling into two parts, electromagnetic simulation to obtain Lorentz force density and ultrasonic simulation to model the ultrasonic wave propagation due to Lorentz force. Electromagnetic simulation can be achieved by the finite element method (FEM) and the analytical method; ultrasonic simulation can be performed with the finite element method (FEM), finitedifference time-domain (FDTD), and the analytical method. Some papers combine the finite element method (FEM) and 
TABLE 1: Summary of methods used for modelling EMAT.

\begin{tabular}{lcccc}
\hline People & \multicolumn{2}{c}{ Electromagnetic simulation } & & \multicolumn{2}{c}{ Ultrasonic simulation } \\
& FEM & Analytical method & FEM & FDTD \\
\hline$[30-33]$ & 0 & & \\
{$[18,22]$} & 0 & & \\
Authors & 0 & & \\
Authors & & 0 & \\
Authors & & 0 & \\
\hline
\end{tabular}

the analytical method to model EMAT; that is, the finite element method (FEM) is used to carry out electromagnetic simulation and the analytical method is to achieve ultrasonic simulation [30-33]. Some papers model EMAT arrays with the finite element method (FEM) for both electromagnetic and ultrasonic simulations, that is, the implicit finite element software COMSOL for the electromagnetic simulation and the explicit finite element software Abaqus for the ultrasonic simulation $[18,22]$. The summary of the state of the art methods used for EMAT modelling is shown in Table 1; the method combining finite element method (FEM) and finitedifference time-domain (FDTD), the method wholly using analytical solutions, and the method combining the analytical method and the finite-difference time-domain (FDTD) method to model EMAT arrays have not been studied before.

The method using finite element method (FEM) and finite-difference time-domain (FDTD) to model EMATs has been reported recently by authors in [34]. This paper proposes a new method using an analytical method and the finite-difference time-domain (FDTD) method to model EMAT arrays. On one hand, the EM analytical approach is used to calculate Lorentz force density for a given coil and a particular DC biased magnet configuration, which then can be fed through to ultrasonic simulations. On the other hand, the finite-difference time-domain (FDTD) method is exploited to describe the ultrasonic wave propagation due to the EM Lorentz force density acting upon the metallic sample.

\section{EMAT Modelling}

An EMAT sensor consists basically of a coil carrying an alternating current, a permanent magnet providing a large static magnetic field, and the test piece, as shown in Figure 1. The coil induces eddy currents $\mathbf{J}$ in the surface layers of the testing material, and the interaction between the static magnetic field $\mathbf{B}$ and eddy currents J produces a Lorentz force density $\mathbf{F}$ based on (1), which in turn generates ultrasound waves propagating within the testing sample:

$$
\mathbf{F}=\mathbf{J} \times \mathbf{B}
$$

In this work, the EMAT modelling consists of two simulations, electromagnetic simulation and ultrasonic simulation. Electromagnetic simulation is used to obtain the eddy current and further the Lorentz force density, which is the link between electromagnetic simulation and ultrasonic simulation.

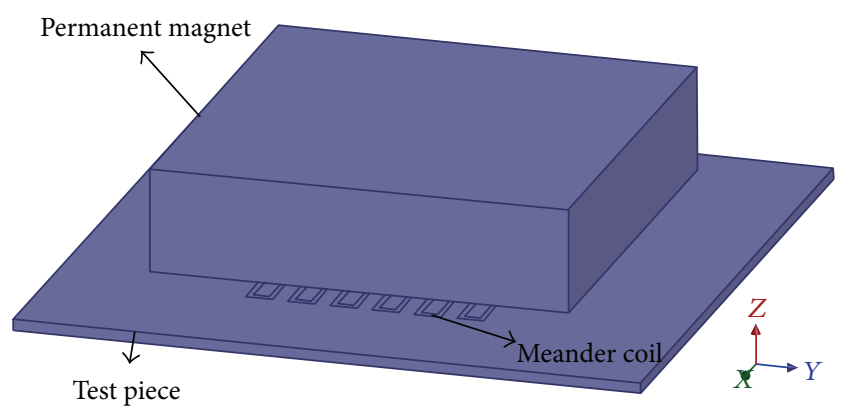

FIGURE 1: The configuration of a typical EMAT.

The testing sample used is a stainless steel plate with a dimension of $1000 \times 1000 \times 80 \mathrm{~mm}^{3}$, and the permanent magnet used is NdFeB35, whose size is $80 \times 80 \times 30 \mathrm{~mm}^{3}$. The meander coil carries an alternating current with the peak of $50 \mathrm{~A}$, the lift-off is $1 \mathrm{~mm}$, the operation frequency is $500 \mathrm{kHz}$, and the skin depth calculated is $0.679 \mathrm{~mm}$. The Rayleigh wave velocity is $3.033 \mathrm{~mm} / \mu$ s in the stainless steel plate used, so the centre-to-centre distance between two adjacent lines of the meander coil is $3.033 \mathrm{~mm}$ to form the constructive interference.

2.1. Electromagnetic Modelling. For electromagnetic calculation, an analytical solution is adapted from the Deeds and Dodd formula to obtain the magnetic vector potential and the eddy current density. Other analytical solutions are available as well $[36,37]$; the analytical solution proposed by Deeds and Dodd is employed as it is of highly satisfactory accuracy and minimum model difference between [35] and authors built.

2.1.1. The Governing Equations. Dodd and Deeds proposed analytical solutions to the circular coil over a layered conductor in [35]. In this work, the geometry used for EM calculation is shown in Figure 2, where a circular coil is placed above the test piece made of stainless steel plate. $r_{1}$ and $r_{2}$ are the inner and outer radius of the circular coil, $h$ is the height of the coil, $l$ is the lift-off distance, and $R$ and $d$ are the length and height of stainless steel plate, respectively.

The governing equations for induced eddy current calculation are

$$
\begin{aligned}
\nabla^{2} \mathbf{A} & =-\mu \mathbf{I}+\mu \sigma \frac{\partial \mathbf{A}}{\partial t}+\mu \epsilon \frac{\partial^{2} \mathbf{A}}{\partial t^{2}}+\mu \nabla\left(\frac{1}{\mu}\right) \times(\nabla \times \mathbf{A}), \\
\mathbf{E} & =-j \omega \mathbf{A}, \\
\mathbf{J} & =\sigma \mathbf{E},
\end{aligned}
$$




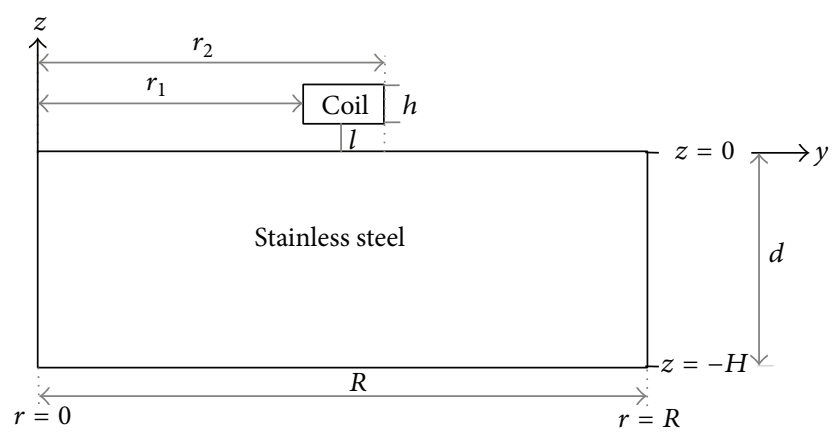

FIgURE 2: The geometry used in [35] for eddy current calculation.

where $\mathbf{A}$ is the vector potential, $\mu, \sigma$, and $\epsilon$ are the permeability, conductivity, and permittivity of the material, respectively, I is the applied current density, $\omega$ is the angular frequency of the applied alternating current, $\mathbf{E}$ is the electric field, and $\mathbf{J}$ is the induced eddy current.

From (2), for eddy current calculation, the main thing is to calculate the vector potential. Dodd and Deeds provided the final analytical solutions to the vector potential calculation as follows [35]:

$$
\begin{aligned}
& \mathbf{A}(r, z)=\mathbf{M} \int_{0}^{\infty} \frac{1}{a^{2}}\left(\int_{a r_{1}}^{a r_{2}} x J_{1}(x) d x\right) J_{1}(a r) \\
& \quad \cdot\left(e^{-a l}-e^{-a(l+h)}\right) \frac{e^{a_{1} z}}{a+a_{1}} d a, \\
& \mathbf{M}=\mu_{0} N \mathbf{I}, \\
& a_{1}=\sqrt{a^{2}+j \omega \mu_{1} \sigma},
\end{aligned}
$$

where $N$ is the turns of the coil, $a$ and $x$ are the integration variables, and $J_{1}(a r)$ and $J_{1}(x)$ are the Bessel functions of first kind. $\mu_{0}$ and $\mu_{1}$ are the permeability of air and metal, respectively.

We build a model to study the analytical solutions to the vector potential problem; the test piece used is stainless steel, and the parameters used are listed in Table 2. By calculating with Matlab, the magnitude distribution of the vector potential $\mathbf{A}$ along the surface of stainless steel $(z=0)$ is shown in Figure 3, where the vector potential is mainly concentrated under the circular coil; the unit of the vector potential is tesla $*$ meter. The blue square in Figure 3 means the maximum vector potential, which shows the distribution of the vector potential is not symmetrical with $r=2.5 \mathrm{~mm}$ because the wire of the circular coil is not straight.

2.1.2. Analytical Method for Meander Coil. In this work, the coil used in EMAT is a meander coil, so the analytical solutions to a straight wire are needed. Based on the analytical solutions proposed by Dodd and Deeds, we proposed an assumption; that is, when the radius of the circular coil is very large, the bent wire of the circular coil can be approximated to a straight wire, and the distribution of the vector potential would be symmetrical. To verify this assumption, we build a model with the same parameters used in Table 2, except
TABLE 2: Parameters used for studying the analytical solutions.

\begin{tabular}{lcc}
\hline Description & Symbol & Value \\
\hline $\begin{array}{l}\text { The length of the stainless } \\
\text { steel }\end{array}$ & $R$ & $5 \mathrm{~mm}$ \\
$\begin{array}{l}\text { The height of the stainless } \\
\text { steel }\end{array}$ & $d$ & $5 \mathrm{~mm}$ \\
$\begin{array}{l}\text { Inside radius of the circular } \\
\text { coil }\end{array}$ & $r_{1}$ & $2.45 \mathrm{~mm}$ \\
$\begin{array}{l}\text { Mean radius of the circular } \\
\text { coil }\end{array}$ & $r_{1}+r_{2}$ & $2.5 \mathrm{~mm}$ \\
$\begin{array}{l}\text { The height of the coil } \\
\begin{array}{l}\text { Permeability of air } \\
\text { Lift-off }\end{array}\end{array}$ & $h$ & $1 \mathrm{~mm}$ \\
$\begin{array}{l}\text { Current density } \\
\text { Outside radius of the } \\
\text { circular coil }\end{array}$ & $\mu_{0}$ & $1.2566 \times 10^{-6} \mathrm{H} / \mathrm{m}$ \\
$\begin{array}{l}\text { Frequency } \\
\text { Conductivity of stainless } \\
\text { steel }\end{array}$ & $\mathbf{I}$ & $1 \mathrm{~mm}$ \\
$\begin{array}{l}\text { Permeability of stainless } \\
\text { steel }\end{array}$ & $r_{2}$ & $1 \mathrm{~A} / \mathrm{m}^{2}$ \\
\hline
\end{tabular}

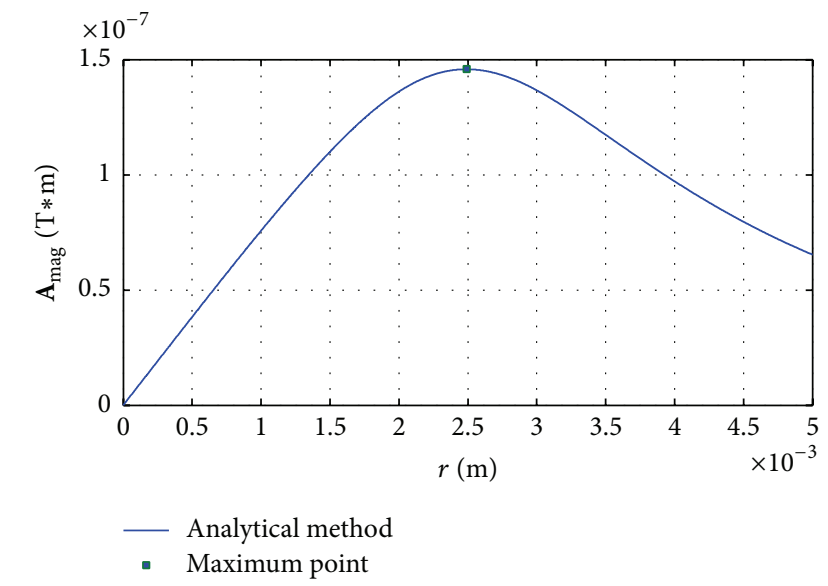

Figure 3: The magnitude distribution of the vector potential A under a circular coil.

that the mean radius of the circular coil is $20.05 \mathrm{~m}$, and the length of the stainless steel sample is $20.1 \mathrm{~m}$. The magnitude distribution of the vector potential A along the surface $(z=$ 0 ) is shown in Figure 4. From this figure, the magnitude distribution is symmetrical with $r=20.05 \mathrm{~m}$, where the wire of the coil is located. This verifies the assumption; that is, when the radius of the spiral coil is very large, the bend wire can be approximated to a straight wire and the solution of Deeds and Dodd can be adapted for this application.

2.1.3. Comparison with FEM at $10 \mathrm{kHz}$. For a straight wire solution, in order to investigate the accuracy of the adapted analytical solutions, the comparison between the analytical solution and the finite element method (FEM) is needed. Maxwell Ansoft is used to construct a model with the same parameters used in Section 2.1.2. The vacuum region to be 


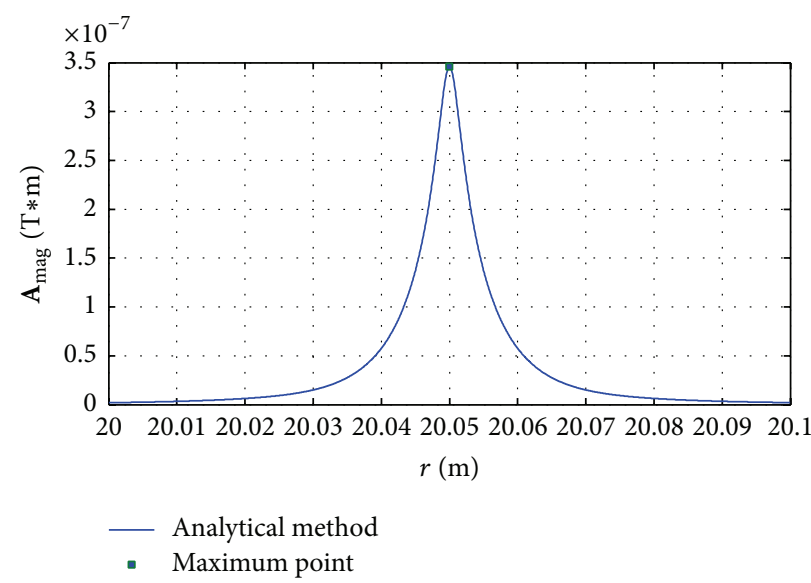

Figure 4: The magnitude distribution of the vector potential $\mathbf{A}$ under a straight wire.

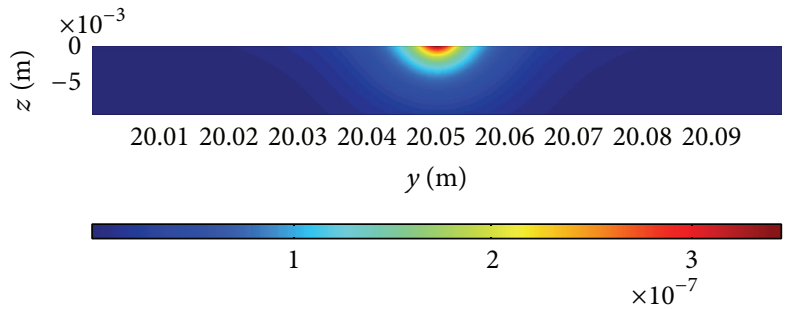

(a) Analytical method

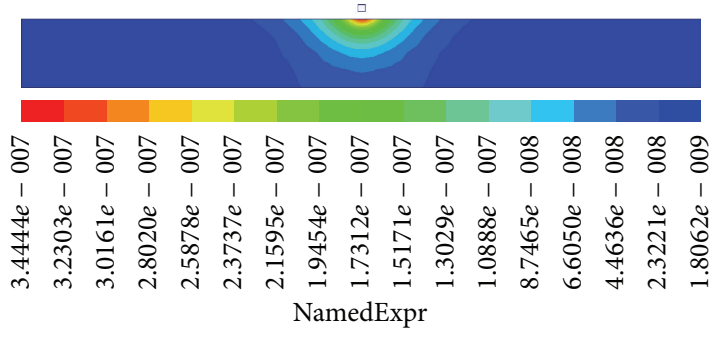

(b) Finite element method (FEM)

FIGURE 5: The magnitude distribution of the vector potential based on different methods.

solved is four times as large as the stainless steel plate; the total number of mesh elements is 43985 . The computation of the FEM solver is based on minimising the energy error, which is as low as $0.068 \%$ in the final iteration (the fifth). The vector distribution on the $y-z$ section of the stainless steel plate with the analytical method and the numerical method is shown in Figures 5(a) and 5(b), respectively. In Figure 5, both the analytical method and the finite element method (FEM) show that the maximum vector potential occurs at the surface of stainless steel plate and decreases along the depth. The magnitude range of the analytical method is consistent with that of the finite element method (FEM), which is from 0 to $3.5 \times 10^{-7}$ tesla $*$ meter.

On the surface of stainless steel $(z=0)$, the distribution of the vector potential is shown in Figure 6, where the magnitude, the real part, and the imaginary part of the vector potential distribution are shown in Figures 6(a), 6(b), and 6(c), respectively. In Figures 6(a) and 6(b), for magnitude and real part distribution, the analytical method and the finite element method are consistent. However, for the imaginary part of the vector potential, as shown in Figure 6(c), the analytical method shows a more accurate result than FEM because the vector potential based on FEM is not approaching zero when $\mathbf{A}$ is away from the wire.

2.1.4. Comparison with FEM at $1 \mathrm{MHz}$. In this part, the analytical solutions at a high operating frequency are studied.
This is because EMAT normally operates at high frequencies and the eddy current is typically limited near the surface. The model used is the same as that in Section 2.1.3, except that the frequency used is $1 \mathrm{MHz}$.

Along the surface of the stainless steel $(z=0)$, the real part distribution of the vector potential based on the analytical method and the finite element method (FEM) is shown in Figure 7. The curve obtained by the analytical method is smoother than that of FEM; that is because of the numerical nature of the FEM; numerical approximation due to finite mesh density and element interpolation are inevitable. With comparison vector potential distribution at $10 \mathrm{kHz}$ and $1 \mathrm{MHz}$, as shown in Figures 6 and 7, respectively, the analytical method is of satisfactory accuracy to describe the electromagnetic properties and is more precise than FEM, in particular for the higher frequency regime.

2.1.5. Analytical EMAT-EM Modelling. The meander coil used in this study has a dimension of $56 \times 34.163 \times$ $0.036 \mathrm{~mm}^{3}$, which is very small compared to the size of the stainless steel plate. In order to improve modelling time, only the area $\left(100 \times 100 \times 2 \mathrm{~mm}^{3}\right)$ where the meander coil mainly has an effect is picked to study the Lorentz force distribution.

As mentioned before, the distribution of the induced eddy current under a straight wire can be obtained by the analytical solutions. For a meander coil, the total induced eddy current is the sum of the induced eddy current caused by each wire 


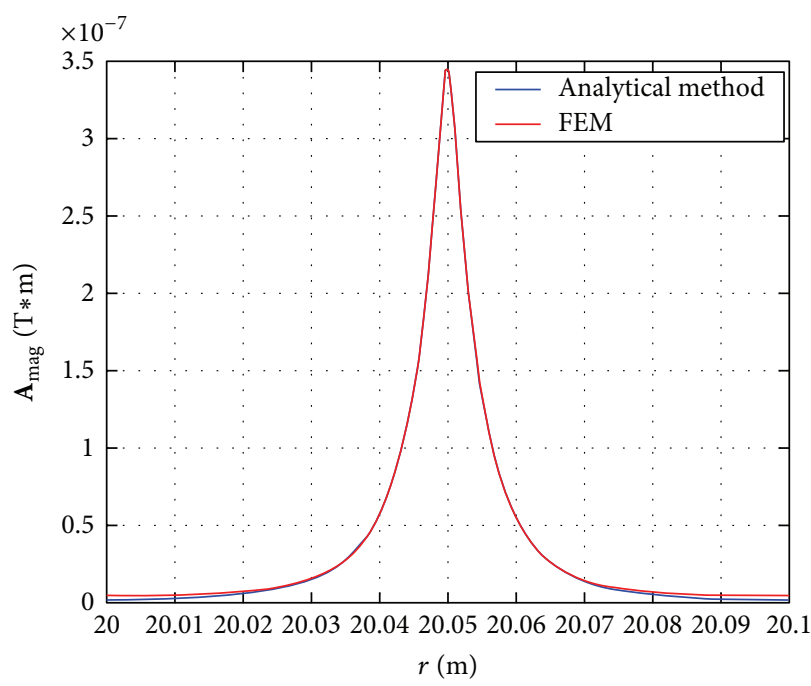

(a)

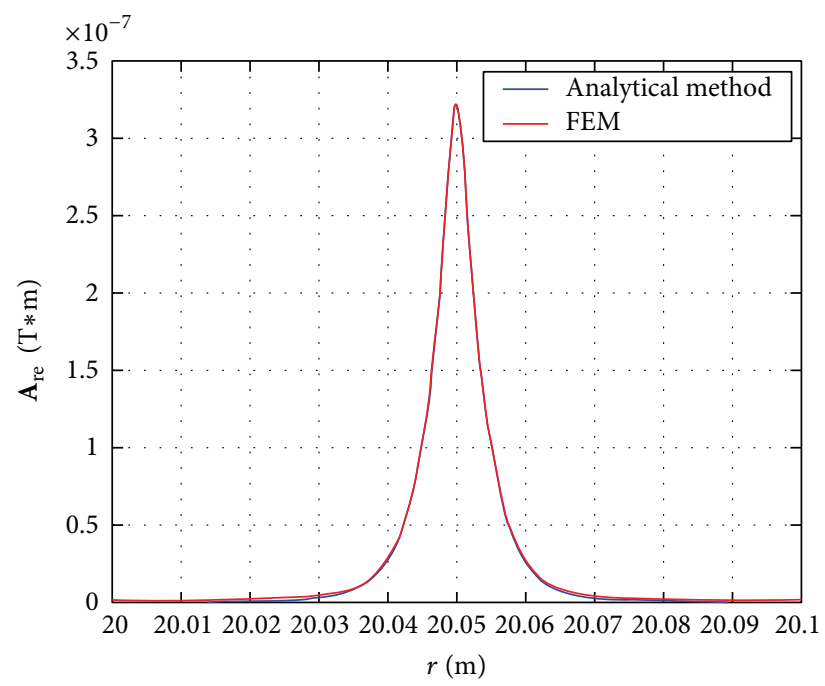

(b)

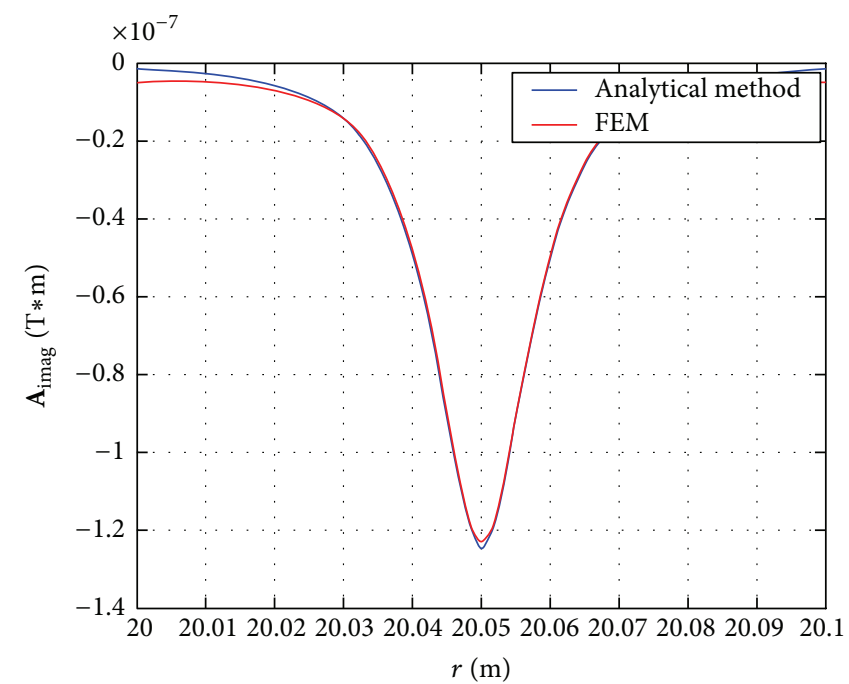

(c)

FIGURE 6: With an operating frequency of $10 \mathrm{kHz}$, the distribution of the vector potential along the surface of stainless steel; (a) the magnitude distribution, (b) the real part distribution, and (c) the imaginary distribution.

segment; the distribution of the induced eddy current on $y-z$ section is shown in Figure 8, where fields between two adjacent wires are opposite due to opposite directions of the alternating currents. In addition, the values of the eddy current under the outmost wires are the largest, because the outmost wires are only affected by the fields on one side.

Along the surface of the stainless steel plate, eddy current distribution is shown in Figure 9(a), which confirms the observation that the amplitude along the outmost wire is the largest. Because there are six pairs of adjacent wires with different current directions, the eddy current plot has 6 crests and 6 troughs. Figure 9(b) shows the distribution of Lorentz force density on the surface of the stainless steel plate. It can be seen that the Lorentz force density on the outmost lines is larger than that on the inner lines; that is because both the maximum magnetic field and the maximum eddy current occur at the places corresponding to the edges of the EMAT sensor.

\subsection{Ultrasonic Modelling}

2.2.1. Governing Equations. Elastodynamic equations are a set of partial differential equations describing how material deforms and becomes internally stressed as shown in the following $[38,39]$ :

$$
\begin{aligned}
\rho(x) \frac{\partial \mathbf{v}_{\mathbf{i}}}{\partial t}(x, t) & =\sum_{j=1}^{d} \frac{\partial \mathbf{T}_{\mathbf{i j}}}{\partial x_{j}}(x, t)+\mathbf{f}_{\mathbf{i}}(x, t), \\
\frac{\partial \mathbf{T}_{\mathbf{i}}}{\partial t} & =\sum_{j=1}^{d} \sum_{i=1}^{d} c_{i j k l}(x) \frac{\partial \mathbf{v}_{\mathbf{k}}}{\partial x_{l}}+\boldsymbol{\theta}_{\mathbf{i j}}(x, t),
\end{aligned}
$$




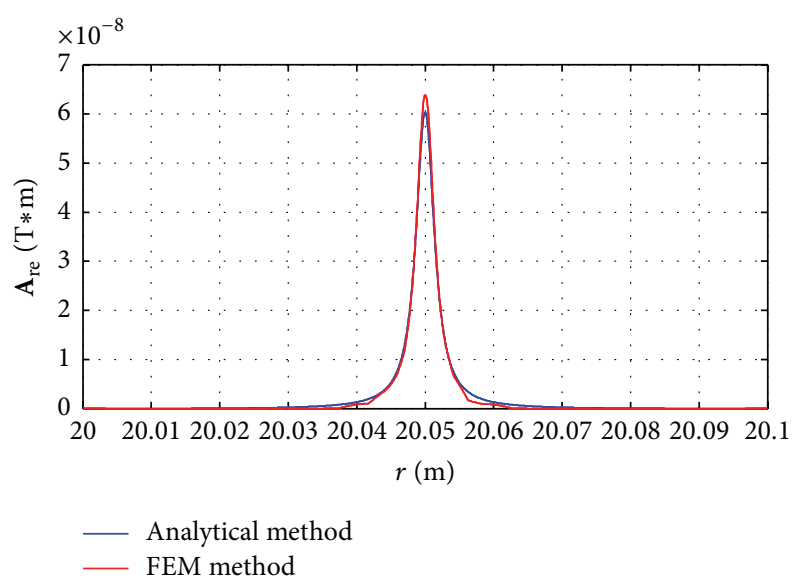

FIGURE 7: With an operating frequency of $1 \mathrm{MHz}$, the real part distribution of the vector potential along the stainless steel surface. Real part of $\mathbf{A}$ under a straight wire.

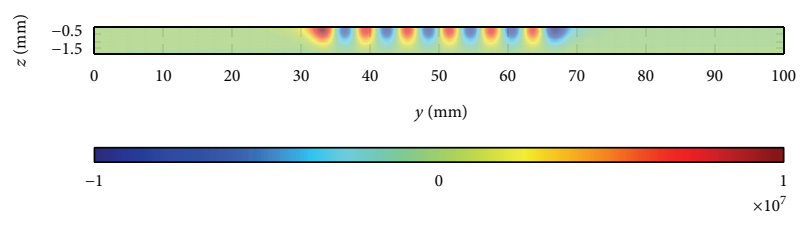

FIGURE 8: The distribution of the induced eddy current based on the analytical method. Real part of the induced eddy current.

where $\rho$ is the mass density and $c_{i j k l}$ is the 4 th stiffness tensor of the testing sample; $\mathbf{f}_{\mathbf{i}}$ and $\boldsymbol{\theta}_{\mathbf{i j}}$ are the force source and strain tensor rate source, respectively. The parameters to be calculated are velocity $\mathbf{v}_{\mathbf{i}}$ and stress tensor $\mathbf{T}_{\mathbf{i}}$. Equation (4) is Newton's Second Law: when a force is applied to a testing sample, stress and deformation are generated, as well as particle displacement. Equation (5) is, based on Hooke's Law, describing the relationship of stress tensor rate and strain tensor rate when deformation occurs.

The finite-difference time-domain (FDTD) method is a numerical method to solve differential equations by discretion of the differential form to the finite-difference form [40]. In this paper, forward difference and centre difference methods are used to calculate the unknown parameters velocity $\mathbf{v}_{\mathbf{i}}$ and stress tensor $\mathbf{T}_{\mathbf{i}}[39]$.

2.2.2. Combination of Electromagnetic Simulation and Ultrasonic Simulation. In this work, Lorentz force density obtained from the electromagnetic model is imported to ultrasonic model to generate ultrasound waves. As shown in Figure 10, the 12 alternating Lorentz force densities are added to the ultrasonic model along the surface. The original $y$ - $z$ cross-section is $1000 * 80 \mathrm{~mm}^{2}$; in order to save modelling geometry and improve modelling time, only the area $\left(400 * 80 \mathrm{~mm}^{2}\right)$ where the EMAT sensor mainly has an effect and surface waves are mainly propagating along is chosen for modelling. Two receivers, R1 and R2, are placed on $(50,79)$ and $(50,77)$, respectively, to inspect the arrival signals.
The propagation of ultrasound waves is shown in Figure 11: at $23 \mu \mathrm{s}$ after firing, the bulk waves, head waves, and surface waves can be identified. Bulk waves contain longitudinal waves and shear waves, which are obliquely propagating into the material; the velocity of the longitudinal waves is larger than that of the shear wave, so the longitudinal wave arrives earlier than the shear wave. Surface waves, which are Rayleigh waves in this work, are propagating along the surface and the subsurface of the material. The velocities of Rayleigh waves and shear waves are slightly different; in most of situations, the velocity of Rayleigh waves is 90 percent of that of the shear waves. So the propagation of Rayleigh waves is slightly delayed than that of shear waves, as shown in Figure 12, where the Rayleigh waves can be identified more clearly at $45 \mu \mathrm{s}$.

The receiving signals from $\mathrm{R} 1$ and $\mathrm{R} 2$ are shown in Figure 13; the first arrival signal is the longitudinal wave arriving at about $25.5 \mu \mathrm{s}$, and the latter arrival signal is the Rayleigh wave at about $49.5 \mu$ s with a large amplitude compared to that of longitudinal waves. The Rayleigh waves can be validated by the distance-of-flight calculation: the distance from R1 and the EMAT sensor is a constant, so the flight distance of the longitudinal waves and the Rayleigh waves should be the same. The velocities of the longitudinal waves and the Rayleigh waves used are $5.9 \mathrm{~mm} / \mu \mathrm{s}$ and $3.033 \mathrm{~mm} / \mu \mathrm{s}$, respectively, and the arrival times of these two waves are $25.5 \mu \mathrm{s}$ and $49.5 \mu \mathrm{s}$, respectively, so the flight distance of the longitudinal waves and the Rayleigh waves calculated is $150.45 \mathrm{~mm}$ and $150.13 \mathrm{~mm}$, which are almost the same. In addition, the amplitude of R1 is slightly larger than that of R2, which confirms that the energy of the Rayleigh waves is mainly distributed in the surface of the material.

The receiving coil used is the same as the transmitting coil; the induced voltage in the receiving coil can be calculated from the receiving velocity fields [41]. In this work, receiving signals calculated are in millivolts. In practical applications, a high power amplifier is essential for EMAT transduction; RITEC RPR4000 and RITEC RAM5000, which are typically used in an EMAT system, are capable of amplifying the receiving signals to volts.

2.3. The Radiation Pattern of EMAT-Rayleigh Waves. Most of the previous works calculate the radiation pattern based on analytical equations [42-44]. In this paper, we define the radiation pattern by combining the FDTD method and the Hilbert transform. For a geometry containing $M * N$ points, each point on the geometry has a time series signal. The max amplitude of the time series signal indicates the arrival time of the signal, and the Hilbert transform is used to calculate the envelope of the signal (6), resulting in identification of the signal arrival time more clearly, as shown in Figure 14:

$$
\begin{aligned}
& h(t)=f(t) * \frac{1}{\pi t}, \\
& z(t)=f(t)+j h(t), \\
& e(t)=\sqrt{f(t)^{2}+h(t)^{2}},
\end{aligned}
$$




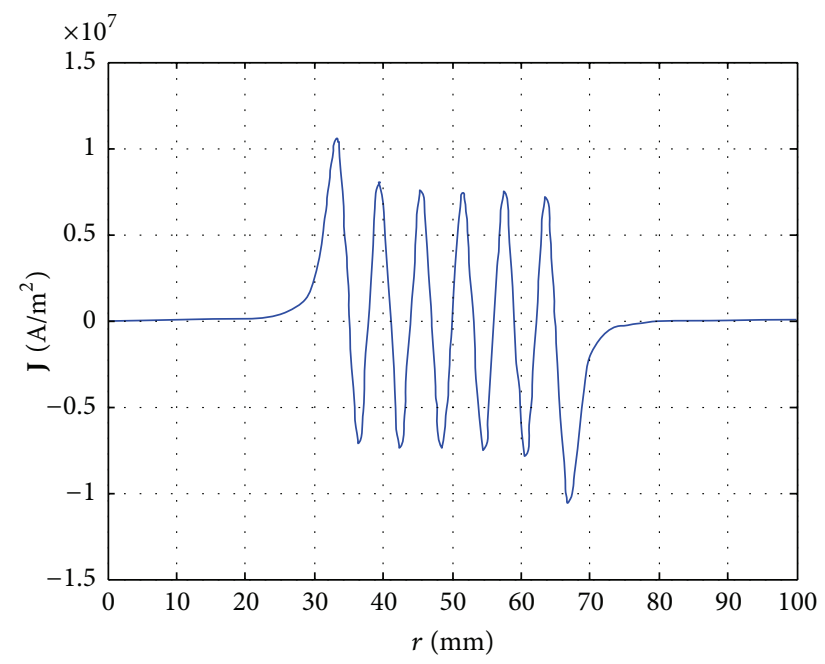

(a)

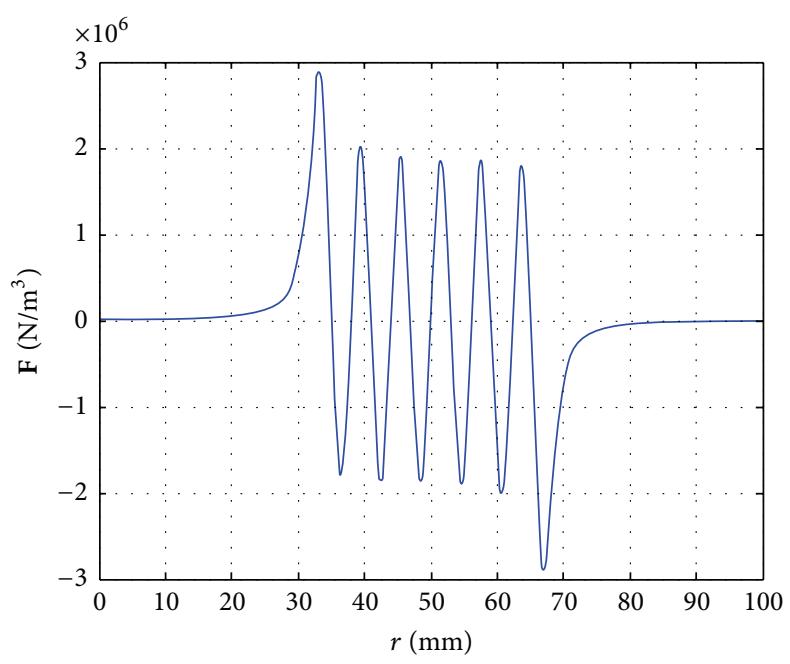

(b)

FIGURE 9: Fields distribution along the surface of the stainless steel plate; (a) the distribution of the induced eddy current and (b) the distribution of Lorentz force density.
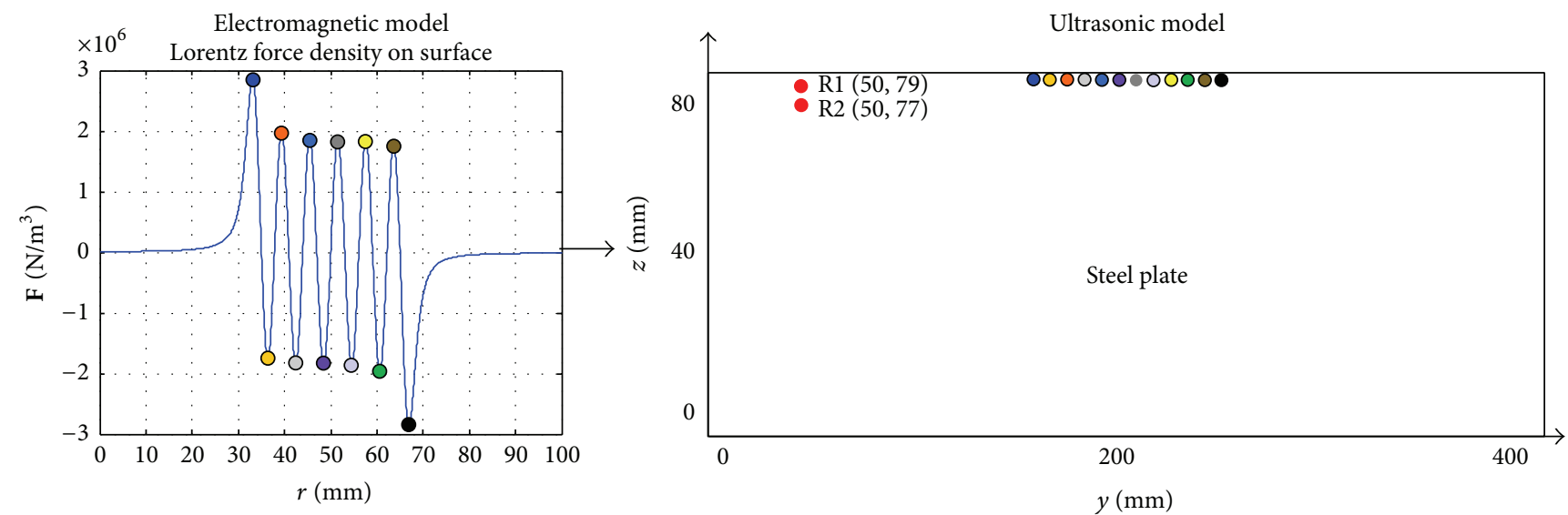

FIGURE 10: Transformation from electromagnetic model to ultrasonic model.

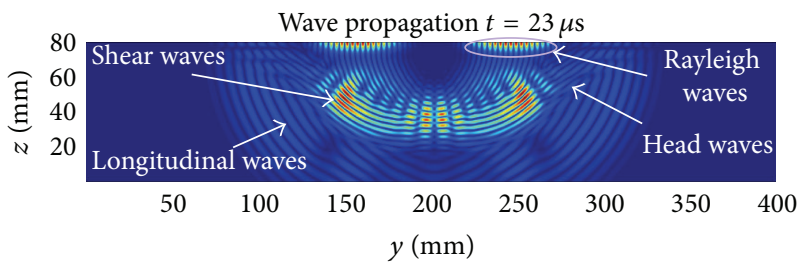

FIGURE 11: Wave propagation at $23 \mu \mathrm{s}$. Four types of waves are produced: longitudinal waves, shear waves, head waves, and Rayleigh waves.

where $f(t)$ is the time series signal, $h(t)$ is the signal after Hilbert transform, $z(t)$ is the analytical signal, and $e(t)$ is the envelope of $z(t)$.

The radiation pattern of the EMAT-Rayleigh waves is shown in Figure 15(a), which shows that Rayleigh waves are mainly distributed along the surface and subsurface of the material; longitudinal and shear waves are propagating

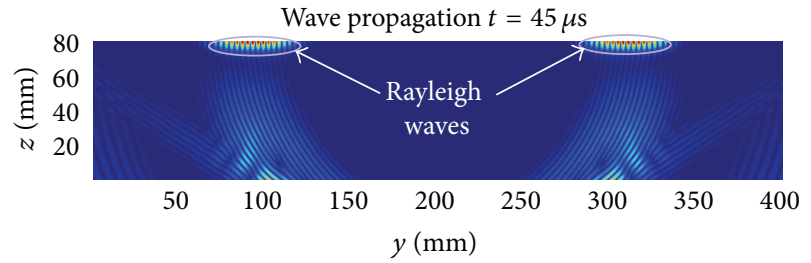

FIGURE 12: Wave propagation at $45 \mu$ s. Rayleigh waves' propagation is slightly delayed compared to the shear waves' propagation.

obliquely into the material, and shear waves have a stronger energy than longitudinal waves. In this work, only Rayleigh waves are of interest; the beam features of Rayleigh waves are studied by means of Figure 15(b).

There are two beam features to be analysed, beam directivity and field distribution along the steering angle. Beam directivity is, at a radial length from the centre of the 

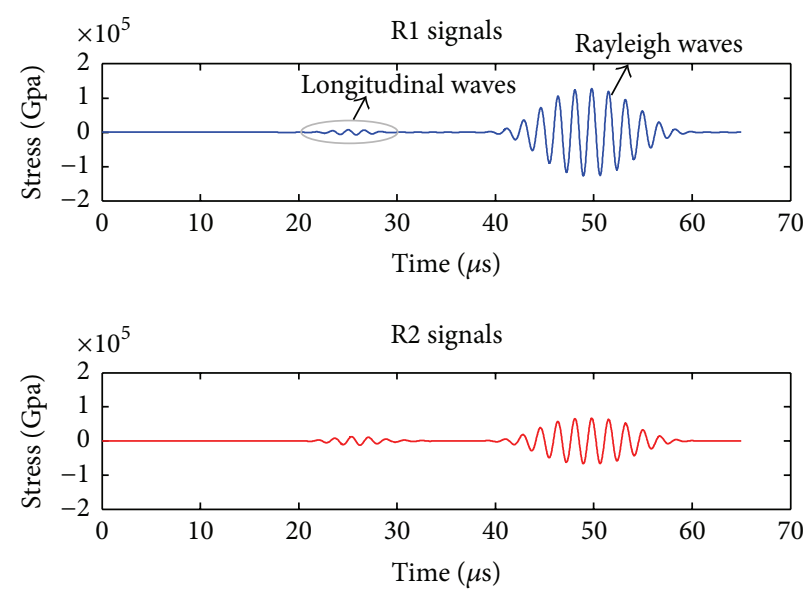

FIGURE 13: Receiving signals from receivers R1 and R2.
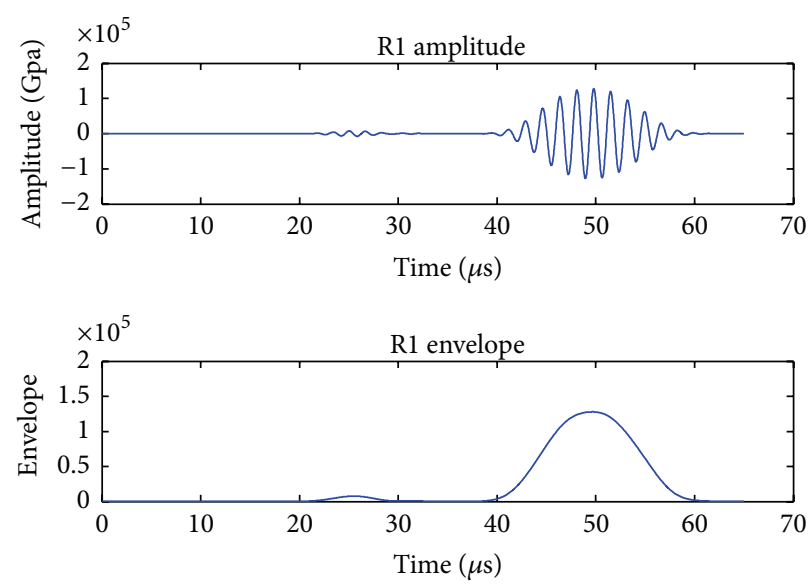

FIGURE 14: The amplitude and envelope of receiving signals from R1.

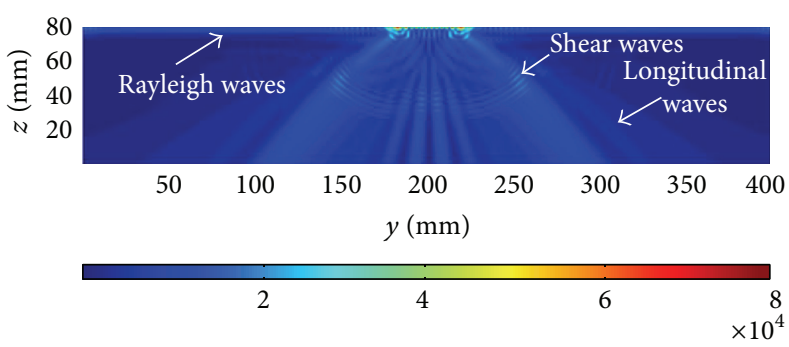

(a) The radiation pattern of EMAT-Rayleigh waves

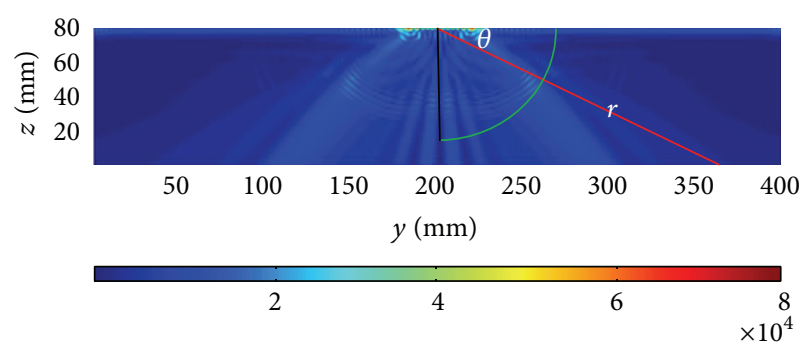

(b) Radiation pattern for beam features

FIGURE 15: The radiation pattern of EMAT-Rayleigh waves for studying beam features.

array, the velocity or pressure distribution, as shown in the green circle arc in Figure 15(b). Field distribution along the steering angle is the velocity or pressure distribution along the steering angle, as shown in the red line in Figure 15(b). $r$ denotes the radial distance from the middle of the sensor and $\theta$ denotes the steering angle; define the steering angle parallel to the surface as $0^{\circ}$; in order to minimise the effects of the longitudinal waves and shear waves, keep the radial distance $r 140 \mathrm{~mm}$ and the steering angle $\theta$ from $0^{\circ}$ to $20^{\circ}$. The beam directivity calculated is shown in Figure 16, where the Rayleigh waves are mainly distributed along the steering angles from $0^{\circ}$ to $2.5^{\circ}$. Because the radial distance $r$ used is $140 \mathrm{~mm}$, the depth $d$ of the Rayleigh waves' energy distribution can be calculated by

$$
d=\tan (\theta) * r
$$

The depth $d$ calculated is $6.1 \mathrm{~mm}$, and the wavelength of the Rayleigh waves used in this work is $6.066 \mathrm{~mm}$, which 

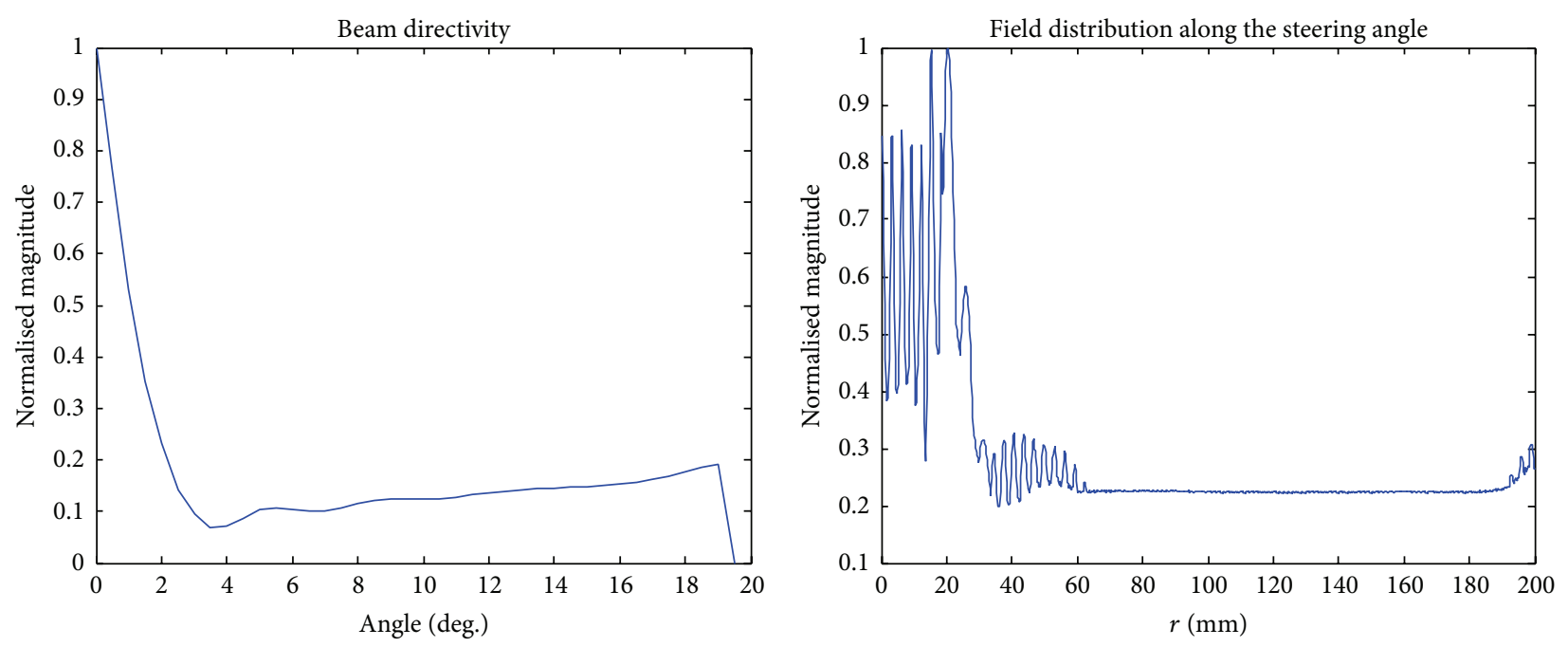

FIGURE 16: The beam directivity and field distribution along the steering angle $0^{\circ}$ of EMAT-Rayleigh waves.

validates that the energy of the Rayleigh waves is mainly distributed within one wavelength of the Rayleigh waves. In addition, when choosing another value of the radial distance $r$, the steering angle $\theta$ changes correspondingly, but the depth $d$ of the Rayleigh waves' energy distribution is a constant.

Another beam feature, field distribution along the steering angle, is studied as well. The radial distance $r$ used is $200 \mathrm{~mm}$ from the centre of EMAT arrays, and the steering angle used is $0^{\circ}$ (along the surface). The field distribution along the steering angle $0^{\circ}$ is shown in Figure 16 and the length period of the maximum magnitudes in this figure is about $17.5 \mathrm{~mm}$, which is consistent with the modelling geometry, where the distance from the centre to the end of the EMAT sensor is $17.1 \mathrm{~mm}$. As a result, the field energy is maximum at the places where the sensor is placed and decreases along the radial distance due to the attenuation of Rayleigh waves.

\section{Conclusion}

A method combining the analytical method for EM simulation and the finite-difference time-domain (FDTD) method for UT simulation to model an EMAT system is proposed. For electromagnetic simulation, analytical solutions to a meander coil are proposed and verified with FEM. By comparing with FEM, the analytical method proposed provides several advantages, especially at high frequencies. The calculated Lorentz force density is used as the excitation source in the ultrasonic simulation, which exploits the finite-difference time-domain (FDTD) method to describe ultrasound wave propagation. The radiation pattern shows that the maximum energy of surface waves is concentrated at the steering angle $0^{\circ}$ and at the position where the sensor is placed.

\section{Conflict of Interests}

The authors declare that there is no conflict of interests regarding the publication of this paper.

\section{References}

[1] M. Cacciola, F. C. Morabito, D. Polimeni, and M. Versaci, "Fuzzy characterization of flawed metallic plates with eddy current tests," Progress in Electromagnetics Research, vol. 72, pp. 241-252, 2007.

[2] D. Lovejoy, Magnetic Particle Inspection: A Practical Guide, Springer, Dordrecht, The Netherlands, 1993.

[3] M. Mahmoudi and S. Y. Tan, "Depth detection of conducting marine mines via eddy-current and current-channeling response," Progress in Electromagnetics Research, vol. 90, pp. 287307, 2009.

[4] M. H. Park, I. S. Kim, and Y. K. Yoon, "Ultrasonic inspection of long steel pipes using Lamb waves," NDT \& E International, vol. 29, no. 1, pp. 13-20, 1996.

[5] W. Yin and A. J. Peyton, "Thickness measurement of nonmagnetic plates using multi-frequency eddy current sensors," NDT \& E International, vol. 40, no. 1, pp. 43-48, 2007.

[6] W. Yin and A. J. Peyton, "Thickness measurement of metallic plates with an electromagnetic sensor using phase signature analysis," IEEE Transactions on Instrumentation and Measurement, vol. 57, no. 8, pp. 1803-1807, 2008.

[7] Q. Zhao, J. N. Hao, and W. L. Yin, "A simulation study of flaw detection for rail sections based on high frequency magnetic induction sensing using the boundary element method," Progress in Electromagnetics Research, vol. 141, pp. 309-325, 2013.

[8] P. Cawley, "Ultrasonic measurements for the quantitative NDE of adhesive joints-potential and challenges," in Proceedings of the IEEE Ultrasonics Symposium, pp. 767-772, Tucson, Ariz, USA, October 1992.

[9] B. Chassignole, D. Villard, M. Dubuget, J.-C. Baboux, and R. El Guerjouma, "Characterization of austenitic stainless steel welds for ultrasonic NDT," Review of Progress in Quantitative Nondestructive Evaluation, vol. 19, pp. 1325-1332, 2000.

[10] B. Drinkwater and P. Cawley, "Measurement of the frequency dependence of the ultrasonic reflection coefficient from thin interface layers and partially contacting interfaces," Ultrasonics, vol. 35, no. 7, pp. 479-488, 1997. 
[11] B. W. Drinkwater and P. D. Wilcox, "Ultrasonic arrays for nondestructive evaluation: a review," NDT \& E International, vol. 39, no. 7, pp. 525-541, 2006.

[12] J. A. Gallego-Juarez, "Piezoelectric ceramics and ultrasonic transducers," Journal of Physics E: Scientific Instruments, vol. 22, no. 10 , pp. 804-816, 1989.

[13] H. Jaffe and D. Berlincourt, "Piezoelectric transducer materials," Proceedings of the IEEE, vol. 53, no. 10, pp. 1372-1386, 1965.

[14] B. Jaffe, Piezoelectric Ceramics, vol. 3, Elsevier, New York, NY, USA, 2012.

[15] D. Caratelli, A. Lay-Ekuakille, and P. Vergallo, "Non-invasivereflectometry-baseddetection of melanoma by piezoelectric micro-needle antenna sensors," Progress in Electromagnetics Research, vol. 135, pp. 91-103, 2013.

[16] R. Ribichini, Modelling of electromagnetic acoustic transducer [Ph.D. thesis], Doctor of Philosophy, Department of Mechanical Engineering, Imperial Colledge London, 2011.

[17] S. Dixon, C. Edwards, and S. B. Palmer, "High accuracy noncontact ultrasonic thickness gauging of aluminium sheet using electromagnetic acoustic transducers," Ultrasonics, vol. 39, no. 6, pp. 445-453, 2001.

[18] R. Dhayalan and K. Balasubramaniam, "A hybrid finite element model for simulation of electromagnetic acoustic transducer (EMAT) based plate waves," NDT \& E International, vol. 43, no. 6, pp. 519-526, 2010.

[19] S. Wang, L. Kang, Z. Li, G. Zhai, and L. Zhang, "3-D modeling and analysis of meander-line-coil surface wave EMATs," Mechatronics, vol. 22, no. 6, pp. 653-660, 2012.

[20] M. Hirao and H. Ogi, EMATs for Science and Industry: Noncontacting Ultrasonic Measurements, Springer, Boston, Mass, USA, 2003.

[21] W. Luo and J. L. Rose, "Guided wave thickness measurement with EMATs," Insight-Non-Destructive Testing and Condition Monitoring, vol. 45, no. 11, pp. 735-739, 2003.

[22] R. Dhayalan and K. Balasubramaniam, "A two-stage finite element model of a meander coil electromagnetic acoustic transducer transmitter," Nondestructive Testing and Evaluation, vol. 26, no. 2, pp. 101-118, 2011.

[23] C. B. Scruby and B. C. Moss, "Non-contact ultrasonic measurements on steel at elevated temperatures," NDT \& E International, vol. 26, no. 4, pp. 177-188, 1993.

[24] R. Edwards, S. Dixon, and X. Jian, "Non-contact ultrasonic characterization of defects using EMATs," in Proceedings of the 31st Annual Review of Progress in Quantitative Nondestructive Evaluation, pp. 1568-1575, Golden, Colo, USA, 2005.

[25] P. J. Latimer and D. T. MacLauchlan, "EMAT probe and technique for weld inspection," Google Patents, 1998.

[26] R. Ludwig, Z. You, and R. Palanisamy, "Numerical simulations of an electromagnetic acoustic transducer-receiver system for NDT applications," IEEE Transactions on Magnetics, vol. 29, no. 3, pp. 2081-2089, 1993.

[27] S. Thomas, S. S. A. Obayya, R. Taneja, and W. Balachandran, "A coupled electromagnetic and mechanical analysis of electromagnetic acoustic transducers," International Journal for Computational Methods in Engineering Science and Mechanics, vol. 10, no. 2, pp. 124-133, 2009.

[28] M. Kaltenbacher, K. Ettinger, R. Lerch, and B. Tittmann, "Finite element analysis of coupled electromagnetic acoustic systems," IEEE Transactions on Magnetics, vol. 35, no. 3, pp. 1610-1613, 1999.
[29] R. Murayama and K. Mizutani, "Conventional electromagnetic acoustic transducer development for optimum Lamb wave modes," Ultrasonics, vol. 40, no. 1-8, pp. 491-495, 2002.

[30] X. Jian, S. Dixon, K. T. V. Grattan, and R. S. Edwards, "A model for pulsed Rayleigh wave and optimal EMAT design," Sensors and Actuators A: Physical, vol. 128, no. 2, pp. 296-304, 2006.

[31] L. Kang, S. Dixon, K. Wang, and J. Dai, "Enhancement of signal amplitude of surface wave EMATs based on 3-D simulation analysis and orthogonal test method," NDT \& E International, vol. 59, pp. 11-17, 2013.

[32] S. Wang, L. Kang, Z. Li, G. Zhai, and L. Zhang, "A novel method for modeling and analysis of meander-line-coil surface wave EMATs," in Life System Modeling and Intelligent Computing, pp. 467-474, Springer, 2010.

[33] S. Wang, Z. Li, L. Kang, and G. Zhai, "Influence of coil parameters on rayleigh waves excited by meander-line coil EMATs," in Intelligent Computing for Sustainable Energy and Environment, vol. 355 of Communications in Computer and Information Science, pp. 94-103, Springer, Berlin, Germany, 2013.

[34] Y. Xie, W. Yin, and A. Peyton, "Quantitative simulation of ultrasonic and EMAT arrays using FEM and FDTD," in Proceedings of the 11th European Conference on Non-Destructive Testing (ECNDT '14), Prague, Czech Republic, 2014.

[35] C. V. Dodd and W. E. Deeds, "Analytical solutions to eddycurrent probe-coil problems," Journal of Applied Physics, vol. 39, no. 6, pp. 2829-2838, 1968.

[36] J. R. Wait, Electromagnetic Waves in Stratified Media: Revised Edition Including Supplemented Material, vol. 3, Elsevier, 2013.

[37] J. R. Wait and K. P. Spies, "Subsurface electromagnetic fields of a line source on a conducting half-space," Radio Science, vol. 6, no. 8-9, pp. 781-786, 1971.

[38] E. Bossy, SimSonic Suite User's Guide for SimSonic3D, 2012.

[39] J. Virieux, "P-SV wave propagation in heterogeneous media: velocity-stress finite-difference method," Geophysics, vol. 51, no. 4, pp. 889-901, 1986.

[40] A. R. Mitchell and D. F. Griffiths, The Finite Difference Method in Partial Differential Equations, John Wiley \& Sons, Chichester, UK, 1980.

[41] X. Jian, S. Dixon, K. Quirk, and K. T. V. Grattan, "Electromagnetic acoustic transducers for in- and out-of plane ultrasonic wave detection," Sensors and Actuators A: Physical, vol. 148, no. 1, pp. 51-56, 2008.

[42] L. Azar, Y. Shi, and S.-C. Wooh, "Beam focusing behavior of linear phased arrays," NDT \& E International, vol. 33, no. 3, pp. 189-198, 2000.

[43] S.-C. Wooh and Y. Shi, "Optimum beam steering of linear phased arrays," Wave Motion, vol. 29, no. 3, pp. 245-265, 1999.

[44] S.-C. Wooh and Y. Shi, "Simulation study of the beam steering characteristics for linear phased arrays," Journal of Nondestructive Evaluation, vol. 18, no. 2, pp. 39-57, 1999. 


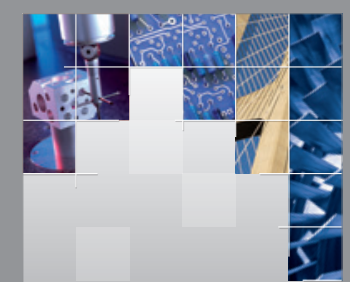

\section{Enfincering}
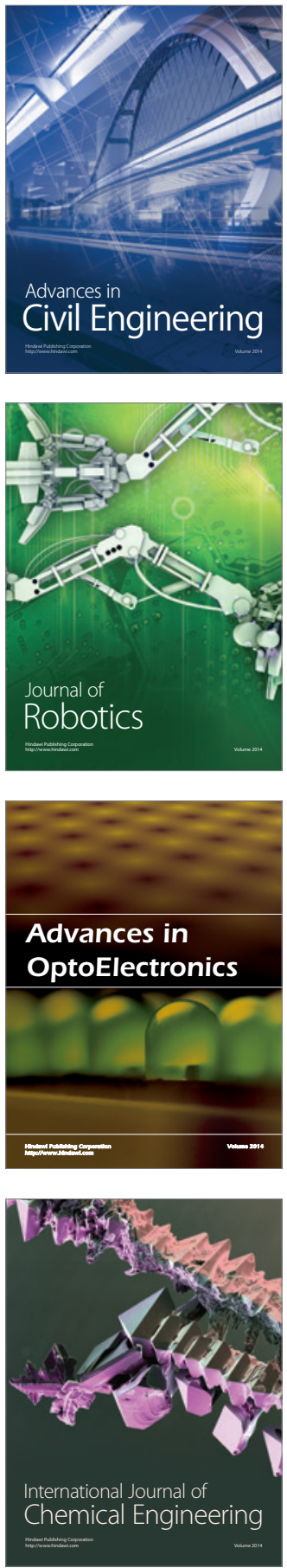

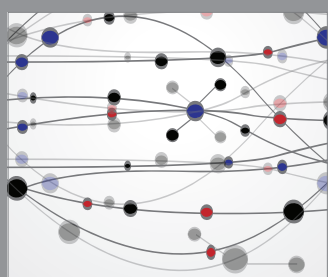

The Scientific World Journal

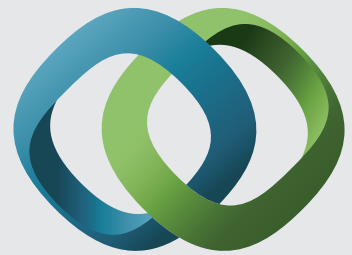

\section{Hindawi}

Submit your manuscripts at

http://www.hindawi.com
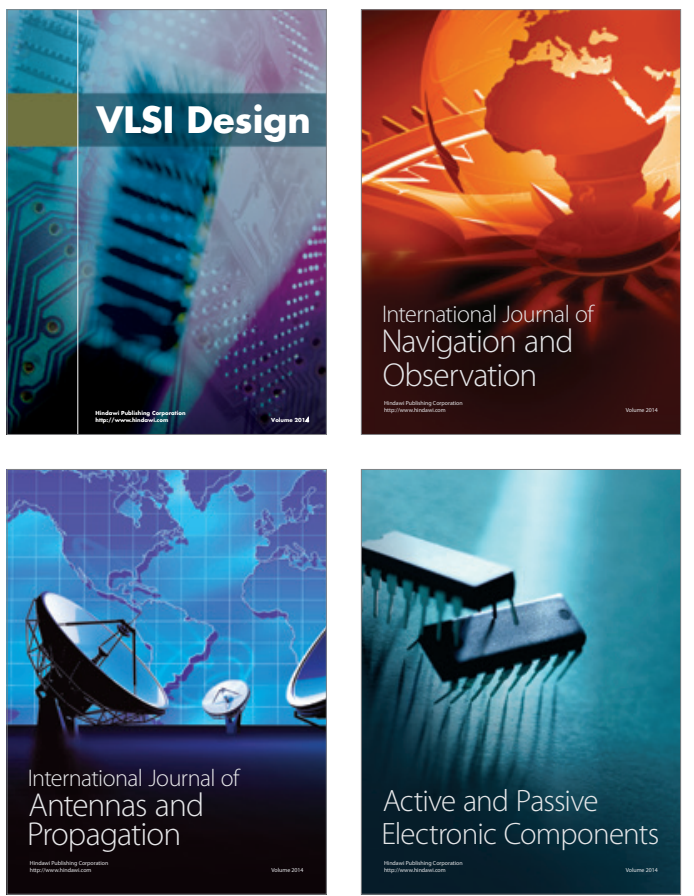
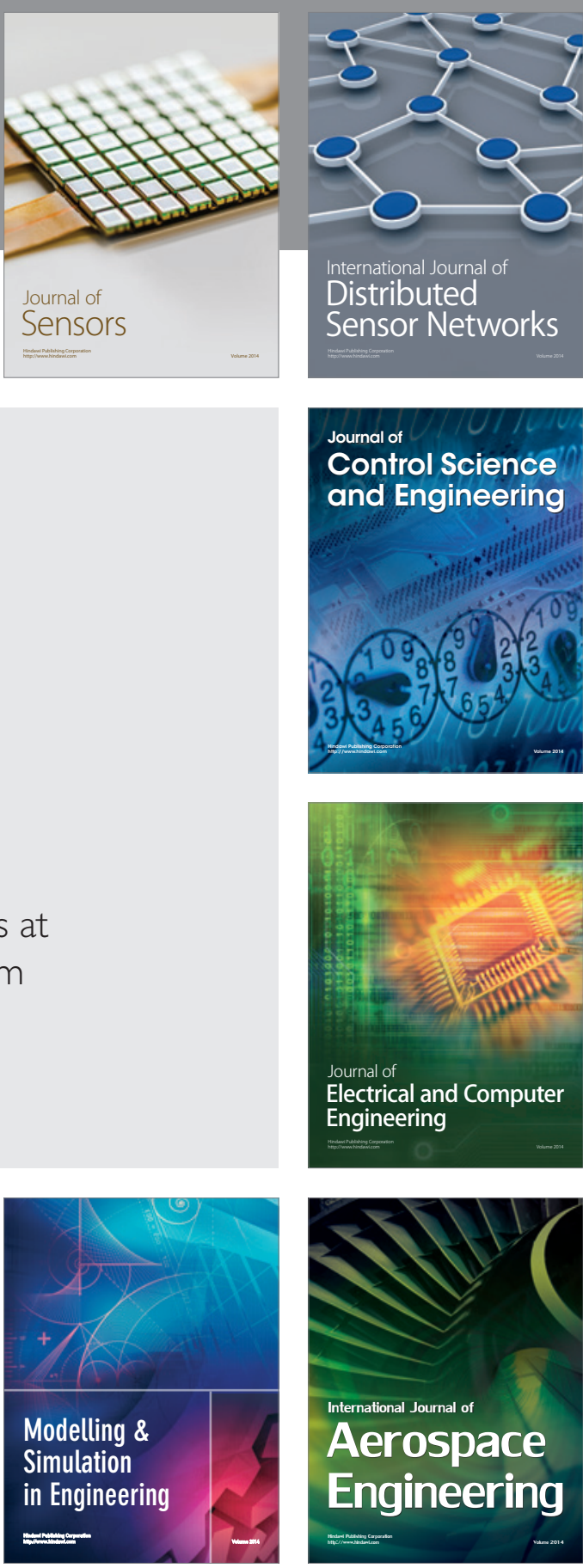

International Journal of

Distributed

Sensor Networks

Journal of

Control Science

and Engineering
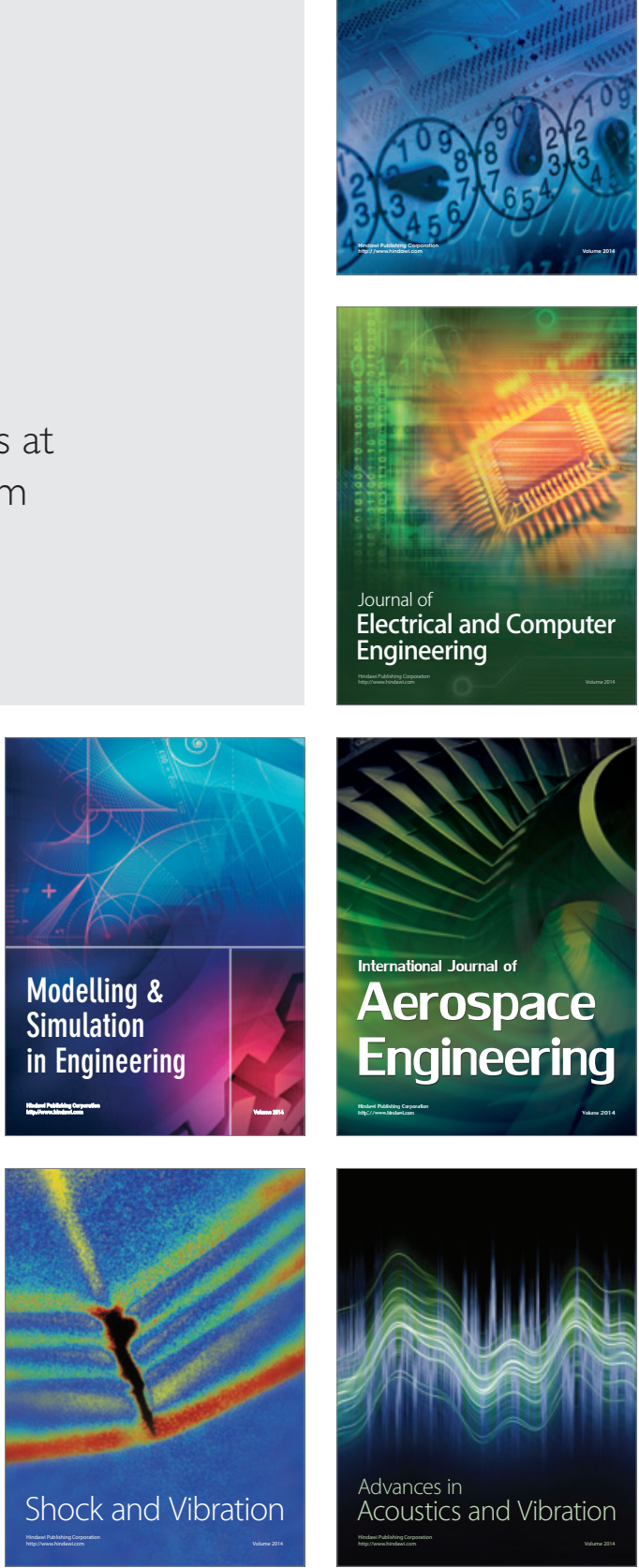